\title{
Could an analysis of mean corpuscular volume help to improve risk stratification in non-anemic patients with acute myocardial infarction?
}

Paweł Franczuk ${ }^{1}$, Maciej Kaczorowski ${ }^{1}$, Karolina Kucharska ${ }^{1}$, Jolanta Franczuk ${ }^{1}$, Krystian Josiak ${ }^{2,3}$, Wojciech Zimoch ${ }^{2,3}$, Michał Kosowski ${ }^{2,3}$, Krzysztof Reczuch ${ }^{2,3}$, Jacek Majda ${ }^{4}$, Waldemar Banasiak ${ }^{2}$, Piotr Ponikowski ${ }^{2,3}$, Ewa A. Jankowska ${ }^{2,5}$

${ }^{1}$ Students' Scientific Association, Laboratory for Applied Research on Cardiovascular System, Department of Heart Diseases, Wroclaw Medical University, Wroclaw, Poland

${ }^{2}$ Cardiology Department, Centre for Heart Diseases, $4{ }^{\text {th }}$ Military Hospital, Wroclaw, Poland

${ }^{3}$ Department of Heart Diseases, Wroclaw Medical University, Wroclaw, Poland

${ }^{4}$ Laboratory Department, $4^{\text {th }}$ Military Hospital, Wroclaw, Poland

${ }^{5}$ Laboratory for Applied Research on Cardiovascular System, Department of Heart Diseases, Wroclaw Medical University, Wroclaw, Poland

\begin{abstract}
Background: Nowadays, when the majority of patients with acute myocardial infarction (AMI) are treated with primary percutaneous coronary intervention and modern pharmacotherapy, risk stratification becomes a challenge. Simple and easily accessible parameters that would help in a better determination of prognosis are needed. The aim of the study was to estimate the prevalence of high mean corpuscular volume ( $M C V$, defined as $M C V>92 \mathrm{fL}$ ) and to establish its prognostic value in non-anemic patients with AMI.
\end{abstract}

Methods: We retrospectively analyzed the data of 248 consecutive non-anemic patients hospitalized due to AMI (median age: 65 [59-76] years, men: 63\%, ST segment elevation myocardial infarction: $31 \%$, and median left ventricular ejection fraction [LVEF]: 50\%).

Results: The prevalence of high MCV was $39 \pm 6 \%$ ( $\pm 95 \%$ confidence interval) in the entire AMI population. High MCV was more prevalent in males, patients with low body mass index, non-diabetics and cigarette smokers (all $p<0.05$ ). During the 180-day follow-up, there were $38(15 \%)$ events, defined as another AMI or death. In a multivariable Cox proportional hazard model, female gender $(p<0.01)$, low LVEF $(p<0.001)$, previous AMI $(p<0.05)$, arterial hypertension $(p<0.05)$, and high $M C V(p<0.001)$ were prognosticators of pre-defined events. Conclusions: In non-anemic patients with AMI, high MCV is an independent prognostic factor of poor outcome defined as another AMI or death. (Cardiol J 2015; 22, 4: 421-427)

Key words: acute myocardial infarction, anemia, mean corpuscular volume, risk stratification

Address for correspondence: Prof. Ewa A. Jankowska, MD, PhD, FESC, Laboratory for Applied Research on Cardiovascular System, Department of Heart Diseases, Wroclaw Medical University, ul. Weigla 5, 53-114 Wrocław, Poland, tel/fax: +48 261660 275, e-mail: ewa.jankowska@umed.wroc.pl

Received: 29.10.2014 Accepted: 09.05.2015 


\section{Introduction}

The determination of prognosis in patients after acute myocardial infarction (AMI) has always been difficult. Nowadays, when the majority of these patients are treated with primary percutaneous coronary intervention (PCI) and modern pharmacotherapy, risk stratification has become even more challenging.

It is obvious that patients after AMI with anemia have greater cardiovascular risk [1-6], whereas in patients without anemia at admission the risk stratification remains equivocal. Easy and cheap parameters that would help in a better determination of prognosis are needed.

It is worth to mention that a complete blood count routinely performed in every patient admitted with AMI includes a simple parameter, namely a red cell mean corpuscular volume (MCV), which potentially could be helpful in risk stratification. High MCV (macrocytosis) is known to be elevated in various conditions, regardless of concomitant anemia [7]. Several studies have addressed associations between high MCV and increased mortality, independently of anemia, in different pathologies, such as chronic kidney disease [8], acute heart failure [9], and in patients after PCI [10]. To the best of our knowledge, a prognostic value of high MCV among patients admitted due to AMI has not yet been analyzed.

Therefore, we performed the study in order to estimate the prevalence of high MCV in non-anemic patients admitted due to AMI and to establish its prognostic value.

\section{Methods}

\section{Study population}

The retrospective study was conducted among consecutive patients hospitalized due to AMI in Center for Heart Diseases, the $4^{\text {th }}$ Military Hospital in Wroclaw, between May and November 2012. There were only two exclusion criteria for the purposes of this study: (1) lack of the patient's written informed consent to participate in the study, and (2) anemia on admission. The diagnosis of AMI was established according to the third universal definition of myocardial infarction introduced by the European Society of Cardiology [11]. The study protocol was approved by the local Ethics Committee, and all subjects gave written informed consent. The study was conducted in accordance with the Declaration of Helsinki.

\section{Clinical and laboratory data}

For the purposes of the following study, we analyzed the following variables in patients admitted due to AMI:

- demographic and anthropometric characteristics: sex, age, body mass index (BMI);

- characteristics of AMI: type of AMI - either ST segment elevation myocardial infarction (STEMI) or non-STEMI (NSTEMI), peak troponin level, primary effective coronary revascularization;

- medical history and co-morbidities: history of previous AMI, stroke, transient ischemic attack, concomitant diabetes mellitus, arterial hypertension, nicotinism, alcohol consumption;

- left ventricular ejection fraction (LVEF) estimated visually with the use of echocardiography during first 48-72 h of hospitalization;

- standard laboratory parameters measured on admission: hemoglobin, estimated glomerular filtration rate (eGFR), gamma-glutamyl transpeptidase (GGTP), low density lipoprotein (LDL) cholesterol;

- medications administered prior to AMI: angiotensin-converting enzyme inhibitors (ACE-I), angiotensin receptor blockers (ARB), antiplatelet drugs, beta-blockers, metformin and statins;

- medications administered during the hospitalization and continued as post-discharge recommendations (post-AMI): ACE-I, ARB, antiplatelet drugs, beta-blockers, metformin and statins;

- MCV measured on admission.

Anemia was defined according to the World Health Organization criteria as hemoglobin $<12 \mathrm{~g} / \mathrm{dL}$ and $<13 \mathrm{~g} / \mathrm{dL}$ in women and men, respectively [12]. MCV was assessed with the use of Sysmex XT-4000i and Sysmex XS-1000i Hematology Analyzers (Sysmex Inc., Kobe, Japan) and calculated according to the formula: $\left[\mathrm{HCT}(\%) / \mathrm{RBC}\left(\times 10^{6}\right)\right.$ $/ \mu \mathrm{L})] \times 10$ [13]. High MCV was defined as MCV > $92 \mathrm{fL}$ (the upper limit of reference ranges in our laboratory, which is also a commonly accepted cut-off for the diagnosis of macrocytosis) [14]. Renal function was assessed using eGFR, calculated from the Modification of Diet in Renal Disease equation [15].

\section{Survival analysis}

The follow-up period of event-free survivors was 180 days. The study endpoint was considered 
another AMI or death. The follow-up information was complete in all cases and was obtained from medical records.

\section{Statistical analyses}

Hemoglobin, eGFR and LDL cholesterol (as continuous variables with a normal distribution) were expressed as means \pm standard deviations. The intergroup differences were tested using the Student's t-test. Age, BMI, peak troponin level, LVEF and GGTP (being continuous variables with a skewed distribution) were expressed as medians with lower and upper quartiles. The intergroup differences were tested using the Mann-Whitney U-test. For further analyses, these variables were $\log$ transformed (a natural logarithm $-\ln$ ) in order to normalize their distribution. Categorical variables were expressed as numbers with percentages. The intergroup differences were tested using the $\chi^{2}$ test.

Clinical determinants of high MCV were established using univariate logistic regression models.

Univariate and multivariable Cox proportional hazard regression models were used to calculate the predictive value of high MCV and other clinical prognosticators for AMI and death. The multivariable model included all variables that had been shown to be significant $(\mathrm{p}<0.05)$ prognosticators of another AMI and death in univariate models.

Kaplan-Meier event-free survival curves were constructed to demonstrate the effect of high MCV on cumulative survival. Differences in event-free survival rates were examined using the log-rank test.

A value of $p<0.05$ was considered statistically significant. All statistical analyses were performed using the Statistica 10 data analysis software system (StatSoft Inc., Tulsa, Oklahoma, United States).

\section{Results}

\section{Baseline characteristics of non-anemic patients admitted due to AMI}

After exclusion of 48 patients with anemia on admission, the study population consisted of 248 patients (Table 1). The median age was 65 (59-76) years, $63 \%$ of patients were men, and NSTEMI was the most common type of AMI (69\%). The mean hemoglobin level was $14.0 \pm 1.3 \mathrm{~g} / \mathrm{dL}$. The mean MCV was $91.4 \pm 5.0 \mathrm{fL}$.

\section{Determinants of high MCV}

$\mathrm{MCV}>92 \mathrm{fL}$ was diagnosed in 96 non-anemic patients admitted due to AMI, which corresponds to a prevalence of $39 \pm 6 \%$ ( $\pm 95 \%$ confidence interval [CI]). None of the studied subjects had MCV $<80 \mathrm{fL}, \mathrm{MCV} \leq 86 \mathrm{fL}$ was found in 37 subjects, $\mathrm{MCV}>86 \mathrm{fL}$ and $\leq 92 \mathrm{fL}$ in $115, \mathrm{MCV}>92 \mathrm{fL}$ and $\leq 98 \mathrm{fL}$ in 76 and MCV $>98 \mathrm{fL}$ in 20 .

Men, patients with low BMI, those without diabetes and cigarette smokers were characterized by greater prevalence of high MCV (Table 1). High MCV was not related to alcohol consumption (Table 1).

\section{Survival analysis}

The proportion of patients surviving the 180-day follow-up free from another AMI or death was $85 \%$ (95\% CI 80-89\%).

In univariate Cox proportional hazard regression models (Table 2), the following variables were related with the increased risk of another AMI or death: female gender, advanced age, low LVEF, previous AMI, arterial hypertension, high LDL cholesterol and high MCV (all p < 0.05).

Female gender, low LVEF, previous MI, arterial hypertension and high MCV (all p < 0.05) remained significant predictors of another AMI or death in patients hospitalized due to AMI also in the multivariable model (Table 2).

The 180-day event-free survival rates were $76 \%$ (95\% CI $68-85 \%$ ) vs. $90 \%$ (95\% CI 85-95\%) in non-anemic patients admitted with AMI with high vs. normal MCV $\left(\chi^{2}=9.56, \mathrm{p}=0.002\right)$ (Fig. 1$)$. The 180-day free of death survival rates were $86 \%$ (95\% CI $78-93 \%$ ) vs. $95 \%$ (95\% CI 91-98\%) in patients with high vs. normal MCV $\left(\chi^{2}=5.50\right.$, $\mathrm{p}=0.02)$. The difference in 180-day event-free survival rates $86 \%$ (95\% CI $75-97 \%$ ) vs. $91 \%$ (95\% CI $86-96 \%)$ in patients with $\mathrm{MCV} \leq 86 \mathrm{fL}$ vs. MCV $>86 \mathrm{fL}$ and $\leq 92 \mathrm{fL}$, respectively, was not significant $\left(\chi^{2}=0.64, \mathrm{p}=0.41\right)$. Neither was the difference $75 \%$ (95\% CI $65-85 \%$ ) vs. $80 \%$ (95\% CI $62-98 \%)$ in patients with MCV $>92 \mathrm{fL}$ and $\leq 98 \mathrm{fL}$ vs. $\mathrm{MCV}>98 \mathrm{fL}\left(\chi^{2}=0.16, \mathrm{p}=0.69\right)$, respectively.

\section{Discussion}

Our study for the first time provides evidence on links between elevated MCV and cardiovascular risk in patients admitted due to AMI. Increased MCV (> $92 \mathrm{fL}$ ) has been associated with a higher incidence of subsequent cardiovascular event or death in these patients during a relatively short follow-up. Most importantly, this relationship has been demonstrated in non-anemic subjects.

$\mathrm{MCV}$ is one of standard blood morphology parameters assessed routinely, however, surpris- 
Cardiology Journal 2015, Vol. 22, No. 4

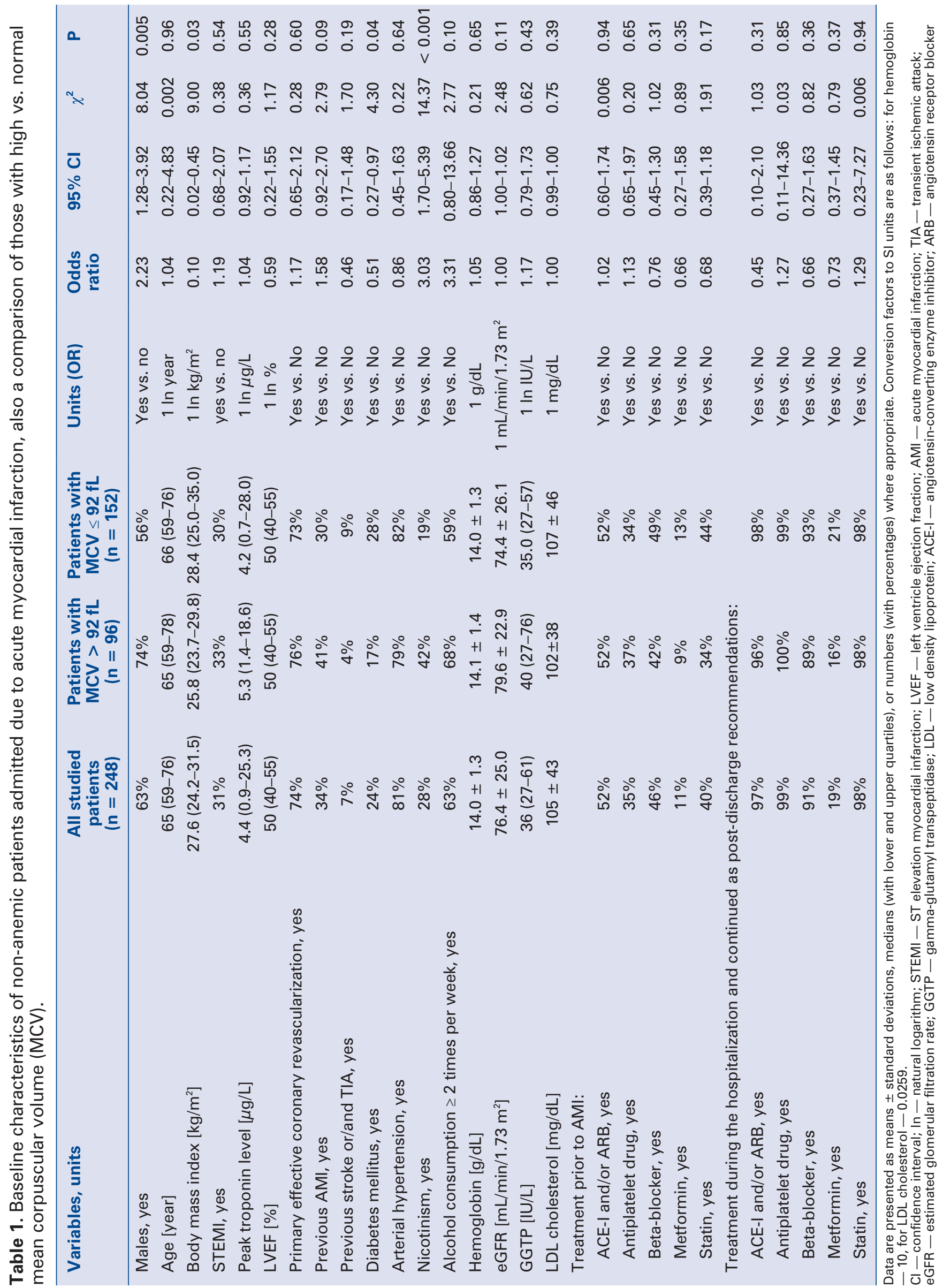




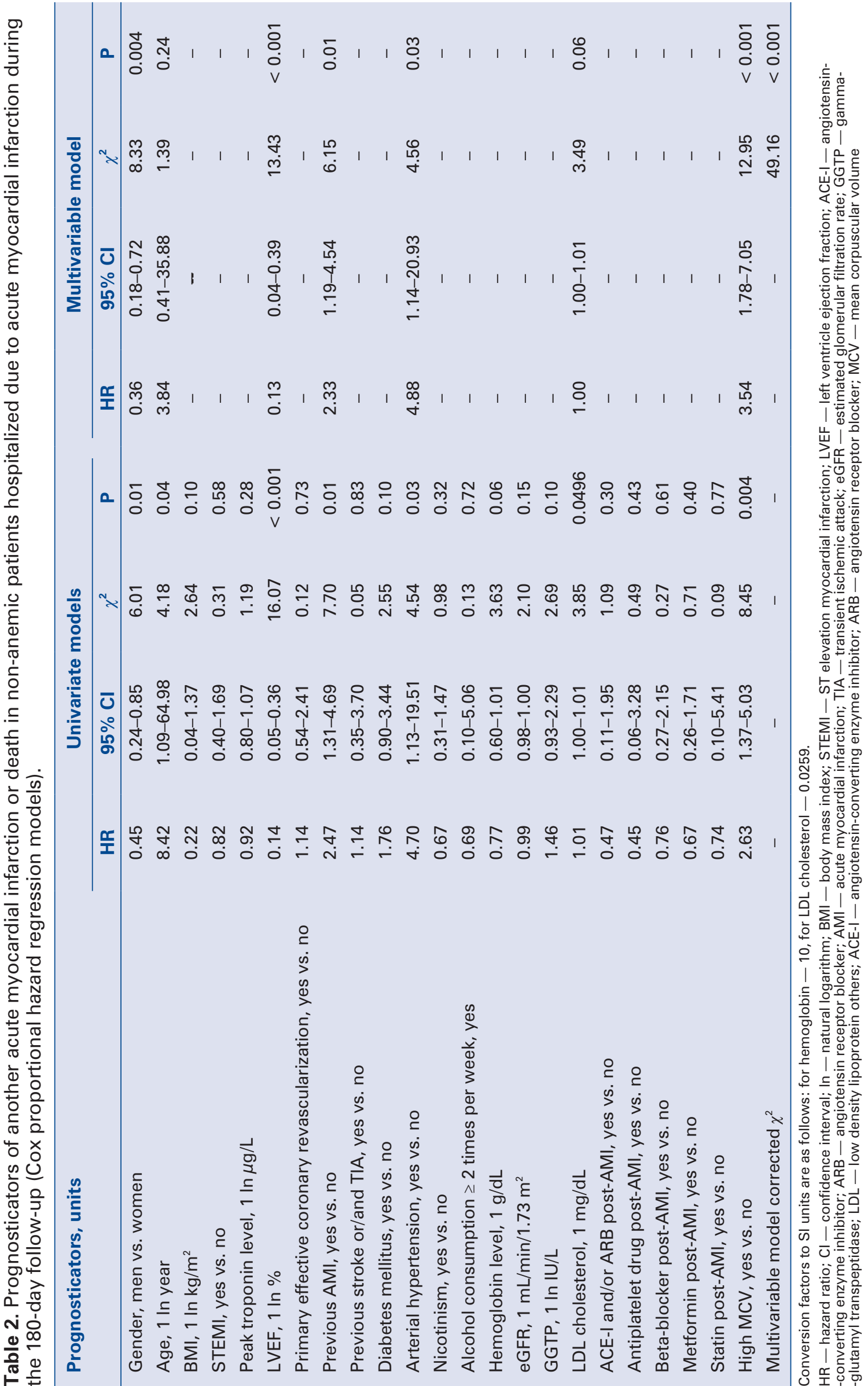




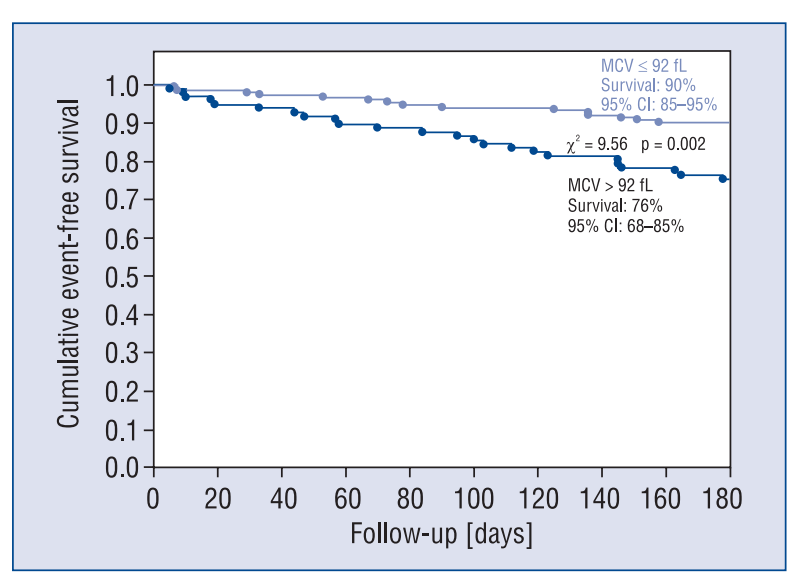

Figure 1. Kaplan-Meier curves reflecting the difference in 180-day event-free survival in patients hospitalized due to acute myocardial infarction with high versus normal mean corpuscular volume (MCV).

ingly receiving not much attention. Macrocytosis is defined as an enlargement of erythrocyte volume, commonly identified as an increase in MCV above $100 \mathrm{fL}$ [16]. However, some studies have demonstrated that lesser deviations in the erythrocyte volume may also indicate pathology and lower cut-off values for high MCV are also used (92 fL) $[14,17]$.

Changes in MCV reflect aberrations on various steps of erythrocyte's life cycle. Depending on the etiology of macrocytosis in non-anemic patients, we may distinguish its megaloblastic and non-megaloblastic forms [16]. Megaloblastic process is caused by impaired DNA synthesis that may be linked to certain drug administration (e.g. metformin, methotrexate, trimethoprim, nitrous oxide), folate or vitamin $\mathrm{B}_{12}$ deficiency $[18,19]$, for instance due to their insufficient supply in food [20, 21]. Non-megaloblastic macrocytes occur when a damaging factor acts after a completed erythropoiesis. Then, it can be caused e.g. by an increased deposition of lipids on the cell membranes in the course of hepatobiliary diseases [19]. Other causes of non-megaloblastic macrocytosis in patients without anemia could be alcoholism [22] or familial macrocytosis [19]. The identification of high MCV does not allow to distinguish these two types of macrocytosis, and we need to acknowledge that the causes of high MCV found in almost 40\% of non-anemic patients admitted due to AMI have not been investigated in our study, which might be considered as a study limitation.

The important and intriguing question is which pathophysiological processes are responsible for the demonstrated relationship between high $\mathrm{MCV}$ and increased cardiovascular risk in subjects without concomitant anemia. Our study was not designed to investigate these pathomechanisms, however some other studies may provide some potential explanations. Possible pathomechanisms can be more or less direct. A direct mechanism would be associated with a hindered flow of larger, less flexible erythrocytes through microcirculation, which is a phenomenon that might contribute to myocardial ischemia [23, 24]. Patients with high MCV commonly develop hyperhomocysteinemia [25], which is known to augment cardiovascular risk [26-28]. Moreover, high MCV is an independent prognosticator of impaired endothelial function measured via flow-mediated dilatation [8], which may be explained by disrupted erythrocyte antioxidative potential in macrocytes and an associated imbalance in global redox homeostasis of the organism [8]. Finally, macrocytosis may reflect a malnutrition, as associations between high $\mathrm{MCV}$, and lower LDL cholesterol levels and lower BMI were reported $[9,10]$.

\section{Conclusions}

In summary, high MCV contributes to cardiovascular risk stratification in non-anemic patients with AMI. After verification of our observations in bigger cohorts and in other clinical contexts, commonly used scales for cardiovascular risk assessment might potentially consider to include MCV to improve the preciseness of risk estimation, which seems to be easily attainable and probably economically advantageous.

\section{Acknowledgements}

This research was financially supported by the statutory grant ST-723 of the Laboratory for Applied Research on Cardiovascular System, Department of Heart Diseases, Wroclaw Medical University, Wroclaw, Poland.

\section{Conflict of interest: None declared}

\section{References}

1. Merono O, Cladellas M, Recasens L et al. In-hospital acquired anemia in acute coronary syndrome. Predictors, in-hospital prognosis and one-year mortality. Rev Esp Cardiol (Engl Ed), 2012; 65: 742-748.

2. Leshem-Rubinow E, Steinvil A, Rogowski O et al. Hemoglobin nonrecovery following acute myocardial infarction is a biomarker of poor outcome: A retrospective database study. Int J Cardiol, 2013; 169: 349-353. 
3. Ang DS, Kao MP, Noman A, Lang CC, Struthers AD. The prognostic significance of early and late anaemia in acute coronary syndrome. QJM, 2012; 105: 445-454.

4. Moura B. [Anemia and prognosis in acute coronary syndromes]. Rev Port Cardiol, 2012; 31: 133-134.

5. Nikolsky E, Aymong ED, Halkin A et al. Impact of anemia in patients with acute myocardial infarction undergoing primary percutaneous coronary intervention: Analysis from the Controlled Abciximab and Device Investigation to Lower Late Angioplasty Complications (CADILLAC) Trial. J Am Coll Cardiol, 2004; 44: 547-553.

6. Kurek T, Lenarczyk R, Kowalczyk J et al. Effect of anemia in high-risk groups of patients with acute myocardial infarction treated with percutaneous coronary intervention. Am J Cardiol, 2010; 105: 611-618.

7. Thong KL, Hanley SA, McBride JA. Clinical significance of a high mean corpuscular volume in nonanemic patients. Can Med Assoc J, 1977; 117: 908-910.

8. Solak Y, Yilmaz MI, Saglam M et al. Mean corpuscular volume is associated with endothelial dysfunction and predicts composite cardiovascular events in patients with chronic kidney disease. Nephrology, 2013; 18: 728-735.

9. Ueda T, Kawakami R, Horii M et al. High mean corpuscular volume is a new indicator of prognosis in acute decompensated heart failure. Circulation J, 2013; 77: 2766-2771.

10. Myojo M, Iwata H, Kohro T et al. Prognostic implication of macrocytosis on adverse outcomes after coronary intervention. Atherosclerosis, 2012; 221: 148-153.

11. Thygesen K, Alpert JS, Jaffe AS et al. Third universal definition of myocardial infarction. Eur Heart J, 2012; 33: 2551-2567.

12. WHO. Haemoglobin concentrations for the diagnosis of anaemia and assessment of severity. Vitamin and Mineral Nutrition Information System. Geneva, World Health Organization, 2011 (WHO/NMH/NHD/MNM/11.1) http://www.who.int/vmnis/indicators/haemoglobin.pdf accessed [17/08/2014].

13. Vajpayee N, Graham S, Bem S. Basic examination of blood and bone marrow. In: McPherson R, Pincus M, Henry J eds. Henry's clinical diagnosis and management by laboratory methods. Saunders Elsevier, Philadelphia 2011: 30.

14. Kabata J, Hellmann A, Podolak-Dawidziak M, Solnica B, Sacha T. Choroby ukladu kriotworczego, Badania laboratoryjne i morfologiczne. In: Gajewski P, Budaj A, Nizankowska-Mogilnicka E eds. Interna Szczeklika: Podrecznik chorob wewnetrznych 2012. Medycyna Praktyczna, Krakow 2012: 1542-1575.
15. Levey AS, Bosch JP, Lewis JB, Greene T, Rogers N, Roth D. A more accurate method to estimate glomerular filtration rate from serum creatinine: A new prediction equation. Modification of Diet in Renal Disease Study Group. Ann Intern Med, 1999; 130: 461-470.

16. Kaferle J, Strzoda CE. Evaluation of macrocytosis. Am Fam Physician, 2009; 79: 203-208.

17. Rumsey SE, Hokin B, Magin PJ, Pond D. Macrocytosis: An Australian general practice perspective. Aust Fam Physician, 2007; 36: 571-572.

18. Aslinia F, Mazza JJ, Yale SH. Megaloblastic anemia and other causes of macrocytosis. Clin Med Res, 2006; 4: 236-241.

19. Veda P. Evaluation of macrocytosis in routine hemograms. Indian J Hematol Blood Transfus, 2013; 29: 26-30.

20. Obeid R, Geisel J, Schorr H, Hubner U, Herrmann W. The impact of vegetarianism on some haematological parameters. Eur J Haematol, 2002; 69: 275-279.

21. Kwok T, Cheng G, Woo J, Lai WK, Pang CP. Independent effect of vitamin B12 deficiency on hematological status in older Chinese vegetarian women. Am J Hematol, 2002; 70: 186-190.

22. Yokoyama T, Saito K, Lwin H, et al. Epidemiological evidence that acetaldehyde plays a significant role in the development of decreased serum folate concentration and elevated mean corpuscular volume in alcohol drinkers. Alcohol Clin Exp Res, 2005; 29: 622-630.

23. Kubota K, Tamura J, Shirakura T et al. The behaviour of red cells in narrow tubes in vitro as a model of the microcirculation. $\mathrm{Br}$ J Haematol, 1996; 94: 266-272.

24. Shiraishi K, Matsuzaki S, Ishida H, Nakazawa H. Impaired erythrocyte deformability and membrane fluidity in alcoholic liver disease: Participation in disturbed hepatic microcirculation. Alcohol Alcohol Suppl, 1993; 1A: 59-64.

25. Haltmayer M, Mueller T, Poelz W. Erythrocyte mean cellular volume and its relation to serum homocysteine, vitamin B12 and folate. Acta Med Austriaca, 2002; 29: 57-60.

26. Veeranna V, Zalawadiya SK, Niraj A et al. Homocysteine and reclassification of cardiovascular disease risk. J Am Coll Cardiol, 2011; 58: 1025-1033.

27. Dworakowska D, Kazimierska E, Weyer-Hepka J et al. Hyperhomocysteinemia complicated by myocardial infarction and portal vein thrombosis: Case report. Pol Arch Med Wewn, 2006; 115: 234-237.

28. Dzielinska Z, Kadziela J, Sitkiewicz D et al. Elevated levels of homocysteine in plasma as a risk factor for coronary artery disease. Pol Arch Med Wewn, 2000; 104: 345-353. 